\title{
Fundamentals of Capillary Electrophoretic Migration and Separation of SDS Proteins in Borate Cross-Linked Dextran Gels
}

\author{
András Guttman,* Csenge Filep, and Barry L. Karger
}

Cite This: Anal. Chem. 2021, 93, 9267-9276

Read Online

ACCESS

Џll Metrics \& More

回 Article Recommendations

ABSTRACT: Recent progress in the development and production of new, innovative protein therapeutics require rapid and adjustable high-resolution bioseparation techniques. Sodium dodecyl sulfate capillary gel electrophoresis (SDS-CGE) using a borate (B) cross-linked dextran (D) separation matrix is widely employed today for rapid consistency analysis of therapeutic proteins in manufacturing and release testing. Transient borate cross-linking of the semirigid dextran polymer chains leads to a high-resolution separation gel for SDS-protein complexes. To understand the migration and separation basis of the $\mathrm{D} / \mathrm{B}$ gel, the present work explores various gel formulations of dextran monomer $(2,5,7.5$, and $10 \%)$ and borate cross-linker (2 and $4 \%$ ) concentrations. Ferguson plots were analyzed for a mixture of protein standards with molecular weights ranging from

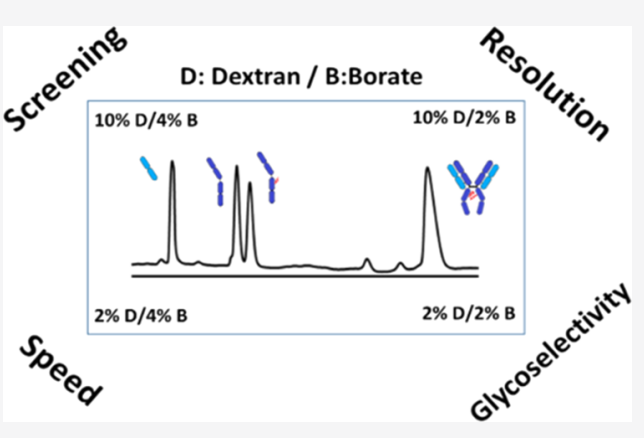
20 to $225 \mathrm{kDa}$, and the resulting nonlinear concave curves pointed to nonclassical sieving behavior. While the $2 \% \mathrm{D} / 4 \% \mathrm{~B}$ gel resulted in the fastest analysis time, the $10 \% \mathrm{D} / 2 \% \mathrm{~B}$ gel was found to produce the greatest separation window, even higher than with the $10 \% \mathrm{D} / 4 \% \mathrm{~B}$ gel, due to a significant increase in the electroosmotic flow of the former composition in the direction opposite to SDS-protein complex migration. The study then focused on SDS-CGE separation of a therapeutic monoclonal antibody and its subunits. A combination of molecular weight and shape selectivity as well as, to a lesser extent, surface charge density differences (due to glycosylation on the heavy chain) influenced migration. Greater molecular weight selectivity occurred for the higher monomer concentration gels, while improved glycoselectivity was obtained using a more dilute gel, even as low as $2 \% \mathrm{D} / 2 \% \mathrm{~B}$. This latter gel took advantage of the dextran-borate-glycoprotein complexation. The study revealed that by modulating the dextran (monomer) and borate (cross-linker) concentration ratios of the sieving matrix, one can optimize the separation for specific biopharmaceutical modalities with excellent column-to-column, run-to-run, and gel-to-gel migration time reproducibilities ( $<0.96 \%$ relative standard deviation (RSD)). The widely used $10 \%$ dextran/4\% borate gel represents a good screening option, which can then be followed by a modified composition, optimized for a specific separation as necessary.

\section{INTRODUCTION}

Various forms of capillary electrophoresis (CE) are widely used as characterization tools in the biopharmaceutical field. ${ }^{1,2}$ Capillary gel electrophoresis (CGE) is of particular importance in the separation and analysis of polyionic macromolecules according to their hydrodynamic volumes, assuming their surface charge densities are constant. ${ }^{3}$ After the early attempts of employing cross-linked polyacrylamide gels, ${ }^{4}$ capillary electrophoresis-based size separation of SDS proteins utilized entangled hydrophilic linear polymer solutions. ${ }^{5-9}$ However, in the new millennia, borate (B) cross-linking-stabilized dextran (D) gels are most often used and represent the industry standard for high-resolution size separation of proteins by capillary electrophoresis. ${ }^{10-12}$

Transient reversible gels of borate cross-linked polyhydroxy macromolecules, featuring instantaneous gelation by mixing, good flow characteristics, and self-healing upon distortionmediated breakage, have long been used for important industrial applications such as fracking. ${ }^{13}$ At neutral $\mathrm{pH}$ values, borate ions form complexes with cis-diol groups, with complex formation involving the $\mathrm{OH}$ groups attached to the adjacent carbon atoms of the polyhydroxy macromolecules (vicinal) or even $\mathrm{OH}$ groups spaced one carbon apart. ${ }^{14}$ Dextran-borate complexes continuously break and reform within the millisecond time scale in a $\mathrm{pH}$, ionic strength, and temperaturedependent manner. ${ }^{15}$ This behavior enables the formation of transient/reversible reticulations, which are similar to chemically cross-linked gels (e.g., polyacrylamide) because of their high-frequency lifetime. ${ }^{16}$

SDS-CGE has been widely used in the biopharmaceutical industry to analyze therapeutic proteins in a simple and robust manner for drug development and commercialization;

Received: April 16, 2021

Accepted: June 10, 2021

Published: June 24, 2021 
however, limited studies have been published to date on the fundamental aspects of this transitionally cross-linked chemical gel separation matrix. ${ }^{17}$ Understanding of the migration and separation behavior of SDS-protein complexes will aid in the optimization of the analysis of specific biopharmaceuticals. This article reports on the effect of the concentration and ratio of the dextran monomer ( $2 \mathrm{MDa}$ ) and borate cross-linker on molecular sieving and separation performance in capillary SDS gel electrophoresis of proteins. A mixture of SDS-bound homologous polypeptides (molecular weight standards) and the intact as well as the subunit forms of a therapeutic monoclonal antibody (mAb) (including its nonglycosylated heavy chain $(\mathrm{ngHC})$ ) were studied under various chemical gel compositions. The interplay between gel sieving and electroosmotic flow (EOF) on separation is explored, along with column performance, to gain a detailed understanding of electromigration and separation. This information can lead to optimized gel compositions for specific separation problems.

\section{EXPERIMENTAL SECTION}

Chemicals. Dextran (2 MDa), boric acid, Tris-base, Ches, sodium dodecyl sulfate, ethylenediaminetetraacetic acid (EDTA), glycerol, 2-mercaptoethanol, iodoacetamide, mesityl oxide, and high-pressure liquid chromatography (HPLC) grade water were from Sigma Aldrich (St. Louis, MO). The $10 \mathrm{kDa}$ internal standard, SDS-MW sizing standard mixture (20-225 $\mathrm{kDa})$, acidic and basic wash solutions $(0.1 \mathrm{M} \mathrm{HCl}$ and $0.1 \mathrm{M}$ $\mathrm{NaOH}$ ), and sample buffer (100 mM Tris-HCl, 1\% SDS, pH 9.0) were from the Sciex SDS-MW analysis assay kit (Brea, CA). Endoglycosidase PNGase F was obtained from Asparia Glycomics (San Sebastian, Spain). The therapeutic monoclonal antibody, omalizumab (Xolair), was a kind gift from the Semmelweis Hospital (Miskolc, Hungary).

Sample Preparation. Ten microliters of the $16 \mathrm{mg} / \mathrm{mL}$ molecular weight sizing standard protein mixture was mixed with $2 \mu \mathrm{L}$ of the internal protein standard $(10 \mathrm{kDa})$ and $5 \mu \mathrm{L}$ of the reducing agent (2-mercaptoethanol), followed by dilution to $80 \mu \mathrm{L}$ with the sample buffer. The therapeutic $\mathrm{mAb}(5 \mu \mathrm{L}$ of $10 \mathrm{mg} / \mathrm{mL})$ was mixed with $2 \mu \mathrm{L}$ of the $5 \mathrm{mg} /$ $\mathrm{mL} 10 \mathrm{kDa}$ protein standard followed by dilution to $75 \mu \mathrm{L}$ with the sample buffer. In the case of the monoclonal antibody subunit analysis, $5 \mu \mathrm{L}$ of 2 -mercaptoethanol and $5 \mu \mathrm{L}$ of 250 $\mathrm{mM}$ iodoacetamide were added to generate the reduced forms. All samples were subjected to gradient temperature denaturation to minimize precipitation. ${ }^{18}$ For $N$-glycan removal, $5 \mu \mathrm{L}$ of $10 \mathrm{mg} / \mathrm{mL}$ reduced and denatured $\mathrm{mAb}$ was mixed with 1.0 $\mu \mathrm{L}$ of PNGase F $(200 \mathrm{mU})$ and incubated at $50{ }^{\circ} \mathrm{C}$ for $60 \mathrm{~min}$. The resulting nonglycosylated heavy chain-containing solution was then mixed with the original (glycosylated) product and used for the experiments.

Separation Matrix Preparation. The buffer systems consisted of 2.0 and $4.0 \%(\mathrm{w} / \mathrm{v})$ boric acid adjusted to $\mathrm{pH}$ 8.0 by Tris base, followed by the addition of EDTA and glycerol in $2 \mathrm{mM}$ and $10 \%(\mathrm{v} / \mathrm{v})$ final concentration, respectively. Then, the appropriate amount of $2 \mathrm{MDa}$ dextran was added to both boric acid containing buffer systems to generate the 2.0, 5.0, 7.5, and $10 \%(\mathrm{w} / \mathrm{v})$ final monomer concentrations. Each mixture was stirred overnight at room temperature to ensure complete dissolution followed by the addition of $0.2 \%(\mathrm{w} / \mathrm{v})$ sodium dodecyl sulfate and slowly stirred for an hour to prevent foaming.

Capillary SDS-Gel Electrophoresis. The PA800 Plus Pharmaceutical Analysis System (Sciex) was used for all separations with UV absorbance detection (214 nm), utilizing $20 \mathrm{~cm}$ effective length $(30 \mathrm{~cm}$ total length) and $50 \mu \mathrm{m}$ ID bare fused-silica capillaries. The applied electric field was $500 \mathrm{~V} / \mathrm{cm}$ in the reversed polarity mode (anode at the detection side), unless otherwise noted. The capillaries were conditioned by rinsing for $3 \mathrm{~min}$ with $0.1 \mathrm{M} \mathrm{NaOH}, 1 \mathrm{~min}$ with $0.1 \mathrm{M} \mathrm{HCl}, 5$ min with HPLC grade water, and finally, for $5 \mathrm{~min}$ with the actual gel-buffer system. The samples were injected electrokinetically by applying $5 \mathrm{kV}$ for $10 \mathrm{~s}$. 32Karat (version 10.1) (Sciex) and PeakFit (version 4.12) were used for data acquisition and analysis. The relative standard deviation (RSD) of the SDS-CGE separation related parameters were as follows: $\alpha_{\text {app }}: 0.1 \%, \alpha_{\text {eff }}: 0.06 \%$, and $R_{s}: 1.94 \%(n=6)$. The average absolute run-to-run $(n=16)$, column-to-column $(n=$ $8)$, and gel-to-gel $(n=3)$ migration time reproducibility, based on all boundary gel compositions, was $0.96 \%$ RSD.

Electroosmotic Flow and Viscosity Measurements. The electroosmotic flow and viscosity of all gel-buffer compositions were measured by injecting $50 \mathrm{mM}$ aqueous mesityl oxide solution and detecting at $254 \mathrm{~nm}$. For electroosmotic flow (EOF) measurements, the separation capillary was conditioned before each separation, as described in the previous paragraph. The applied electric potential was $15 \mathrm{kV}$ at $25{ }^{\circ} \mathrm{C}$ in the normal polarity mode (anode at the injection side). The EOF marker (mesityl oxide) was electrokinetically injected by $5 \mathrm{kV}$ for $20 \mathrm{~s}$ from the anodic end. All other separation parameters were the same as those used for sample analysis. For viscosity measurements, the mesityl oxide was hydrodynamically injected by 5 psi for $5 \mathrm{~s}$ at the inlet side, then the capillary was pressurized by $20 \mathrm{psi}$ (forward) without the application of the electric field. The viscosity marker advanced to the detection window, and the recorded migration time was used for viscosity calculation, based on the Hagen-Poiseuille equation. ${ }^{19}$ The relative standard deviation (RSD, $n=6$ ) of the physical parameter measurements were as follows: $\eta$ : $0.4 \%$, current: $0.07 \%$, and $\mu_{\mathrm{EOF}}: 0.76 \%$.

\section{RESULTS AND DISCUSSION}

As is well known in polymer science, dramatic changes occur when cross-linking takes place with linear polymers leading to a gel structure. ${ }^{20}$ This phenomenon is seen when dextran is dissolved in a solution of boric acid at neutral $\mathrm{pH}$, i.e., addition of borate, allowing cross-linking, significantly changed the matrix structure, as seen in Figure 1. The present study explores the CE electromigration of SDS-protein complexes in borate cross-linked dextran gels under various monomer (D)/cross-linker (B) concentrations. First, the column boundary conditions were defined for the range of gel compositions in terms of ease of gel replacement (viscosity) and conductivity (ionic strength of the separation medium). Once established, some key physical (viscosity and current) and electrokinetic (electroosmotic flow) parameters were measured. Then, Ferguson plots of a mixture of protein sizing standards as well as the intact and subunit forms of a therapeutic $\mathrm{mAb}$ were analyzed for all gel compositions to examine the basic molecular sieving behavior for the solutes. This was followed by an examination of capillary gel electrophoretic separation of the protein standards as well as the intact $\mathrm{mAb}$ and its subunits.

Dextran/Borate Structural Characteristics. Dextran polymers are semirigid chains, composed of $\alpha 1$-6-linked glucose units, in which case six-member borate complexes 


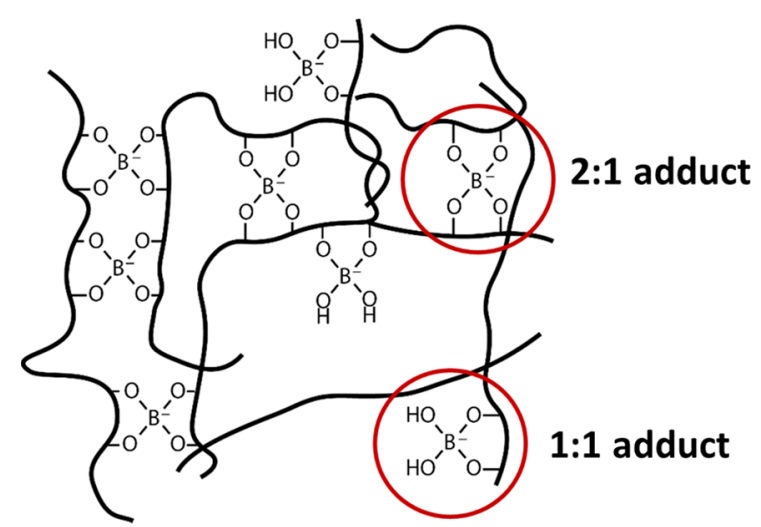

Figure 1. Schematic representation of the borate cross-link-stabilized dextran gel with interchain (2:1) and intrachain (1:1) adducts, highlighted by the circles.

can be assembled in $1: 1$ or $2: 1$ ratios. $^{21}$ As a result, intra- $(1: 1)$ and interchain $(2: 1)$ adducts are formed, the latter providing the necessary cross-linking for the chemical gel formation, as shown in Figure 1. As large SDS-protein complexes electromigrate through such matrices, the high-frequency cross-linking structure can distort and self-heal after the molecules pass through, thus restoring the original sieving capability. $^{22}$ This transient behavior of the gel allows reasonable pressure drop replenishment of the separation matrix in the capillary and also alleviates any local EOFmediated bubble formation, as it was previously found to be problematic for permanently cross-linked polyacrylamide gels in narrow bore capillaries. ${ }^{23}$ At constant dextran concentrations, the borate level in the liquid medium controls the extent of the formation of intrachain (1:1) and interchain (2:1) bonds, thus influencing the level of cross-linking. Elevating the borate concentration increases the number of interchain bonds (cross-linking), resulting in decreasing average pore size and a more structured mesh. However, the presence of excess boric acid in the liquid buffer can lead to saturation of the 2:1 bonds, and the concomitantly increased number of intrachain (1:1) adducts can cause repulsion between the dextran chains, resulting in larger pore sizes. ${ }^{24}$ Finally, as the borate complexed gels are negatively charged in both adduct types, the dextran fibers may tend to align in the high electric field of $\mathrm{CE}$, resulting in more structured reticulation (decreased configuration entropy), similar to that previously suggested for agarose, which is also negatively charged, in slab gel electrophoresis. $^{25}$

Dextran/Borate Composition Limits in the Capillary Electrophoretic System. The composition limits of monomer and cross-linker concentrations for the specific commercial capillary column dimensions (20 cm effective/30 $\mathrm{cm}$ total length and $50 \mu \mathrm{m}$ ID) were chosen based on initial experimental studies. For the $2 \mathrm{MDa}$ dextran, above $10 \%$ (w/ v) concentration, the viscosity of the sieving matrix was too high for simple gel replacement in the narrow bore tubing, considering the upper pressure limit of the capillary electrophoretic instrument. On the other hand, below $2 \%$, the sieving capacity in the molecular weight range of interest (20-225 $\mathrm{kDa}$ ) was poor. For borate, increasing the concentration above $4 \%(\mathrm{w} / \mathrm{v})$ significantly increased the electric current for the given electric field $(500 \mathrm{~V} / \mathrm{cm})$ of the $30 \mathrm{~cm}$ column length, thus leading to undesirable high Joule heating; below $2 \%$ boric acid concentration, the countercurrent EOF in the bare fusedsilica capillary was too high to obtain reasonable analysis times. Thus, the ranges studied were $2-10 \%$ for the dextran monomer in conjunction with the 2 and $4 \%$ borate crosslinker.

Physical and Electrokinetic Parameters. Gel viscosities were measured using the $\mathrm{CE}$ instrument, as described in the Experimental Section. For simplicity, Table 1 presents the widest range of dextran concentrations ( 2 and $10 \%$ ) for the two borate concentrations tested ( 2 and 4\%); other compositions had intermediate viscosities. The table shows that the $10 \% \mathrm{D} / 4 \% \mathrm{~B}$ chemical gel had the greatest viscosity, probably due to the high number of $2: 1$ dextran/borate adducts, as illustrated in Figure 1, providing extensive crosslinking to the dextran chains. With the use of less boric acid in the separation matrix $(10 \% \mathrm{D} / 2 \% \mathrm{~B})$, the decreased crosslinking resulted in a lower viscosity. The viscosity of the $2 \% \mathrm{D} /$ $2 \% \mathrm{~B}$ gel was slightly lower than that of the $2 \% \mathrm{D} / 4 \% \mathrm{~B}$ gel and both were almost 10 -fold lower than the $10 \%$ dextran gels (Table 1 ). In the $2 \%$ dextran gels, the lower number of polymer strands likely led to a large portion of mainly 2:1 interchain complexation, especially with the $2 \%$ borate concentration. In contrast, with an increased amount of borate (4\%) in the low concentration dextran gel, the number of 1:1 adducts likely increased, enhancing the charge density on the non-cross-linked dextran strands and causing repulsion of the negatively charged intrachain complexes. This chain repulsion could lead to larger pores and a less structured polymer matrix (increased configuration entropy). ${ }^{26}$

Another important physical parameter listed in Table 1 is the current, measured with the electric field at $500 \mathrm{~V} / \mathrm{cm}$. The current was clearly higher for the gels with $4 \% \mathrm{~B}$, relative to those for $2 \% \mathrm{~B}$. The largest current was found for the low dextran/high boric acid composition of $2 \% \mathrm{D} / 4 \% \mathrm{~B}$, due to the number of released protons upon the dextran/borate complexation reaction ${ }^{27}$ in the lower viscosity matrix. The lowest current was measured with the high monomer/low crosslinker-containing gel of $10 \% \mathrm{D} / 2 \% \mathrm{~B}$. Other currents for the intermediate monomer compositions were at levels between the ranges shown in Table 1.

Since industry standard dextran-based gels are used in uncoated capillaries, bare fused-silica columns are employed in all experiments. Therefore, it is important to determine the electroosmotic flow (EOF) for each gel column type, as EOF is

Table 1. Physical and Electrokinetic Parameters for the Widest Range Monomer/Cross-Linker Concentrations for Dextran/ Borate Gel Compositions ${ }^{a}$

$\begin{array}{cc}\text { physical/electrokinetic parameters } & 10 \% \mathrm{D} / 2 \% \mathrm{~B} \\ \eta[\mathrm{mPa} \cdot \mathrm{s}] & 85 \\ \text { current }[\mu \mathrm{A}] & 22 \\ \mu_{\mathrm{EOF}}\left(\times 10^{-9}\right)\left[\mathrm{m}^{2} /(\mathrm{V} \mathrm{s})\right] & -0.92\end{array}$

${ }^{a} \mathrm{D}$-dextran, B-boric acid, $\eta$ - viscosity, and $\mu_{\mathrm{EOF}}$ - electroosmotic flow.

$\begin{array}{ccc}10 \% \mathrm{D} / 4 \% \mathrm{~B} & 2 \% \mathrm{D} / 2 \% \mathrm{~B} & 2 \% \mathrm{D} / 4 \% \mathrm{~B} \\ 100 & 11 & 12 \\ 27 & 30 & 36 \\ -0.18 & -4.92 & -2.29\end{array}$



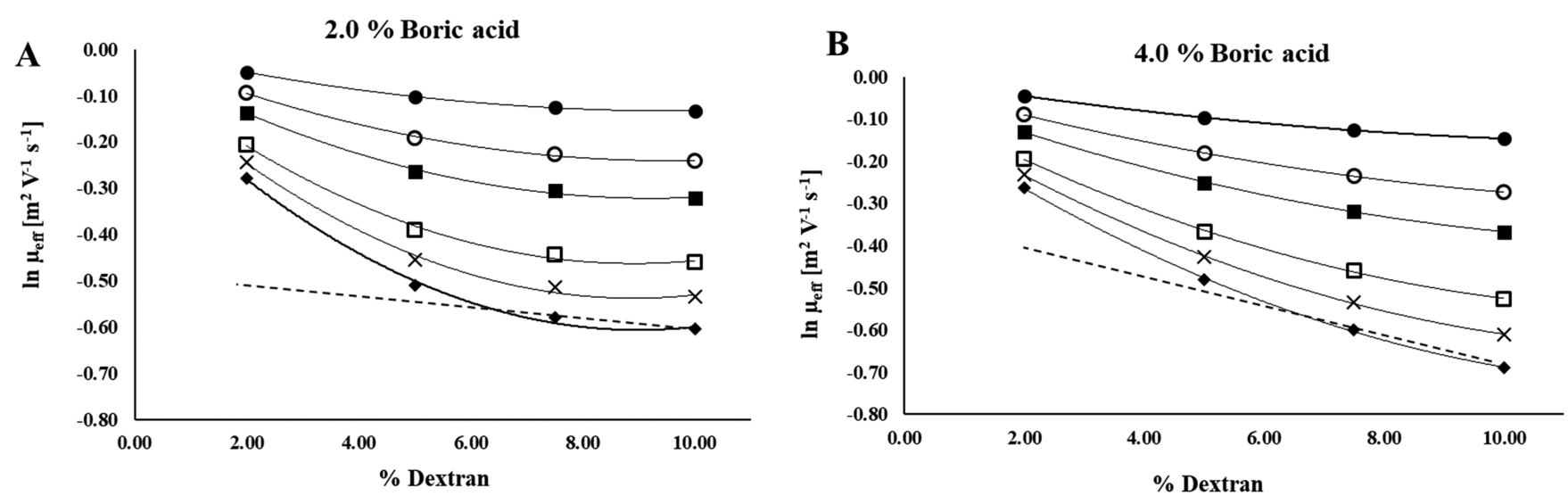

Figure 2. Ferguson plots of the EOF-corrected logarithmic relative electrophoretic mobilities of the molecular weight sizing proteins as a function of monomer (dextran) concentrations with (A) $2.0 \%$ and (B) $4.0 \%$ boric acid cross-linker. Symbols: $20 \mathrm{kDa}, \bigcirc 35 \mathrm{kDa}, \square 0 \mathrm{kDa}, \square 100 \mathrm{kDa}, \mathrm{x}$ $150 \mathrm{kDa}$, and $225 \mathrm{kDa}$ protein standard. The dotted lines guide the eye for the imaginary slopes between the two highest dextran concentration matrices $(7.5$ and $10.0 \%)$.

known to affect the overall separation. ${ }^{28}$ The EOF values for the four widest range gel concentrations at $500 \mathrm{~V} / \mathrm{cm}$ are listed in Table 1. At the $\mathrm{pH}$ of the SDS-CGE buffer, the electroosmotic flow was cathodic; therefore, the EOF was countercurrent to the electromigration of the negatively charged SDS-protein complexes (anodic migration). Thus, the value of the electroosmotic flow mobility $\left(\mu_{\mathrm{EOF}}\right)$ was added to the apparent mobility $\left(\mu_{\text {app }}\right)$ to determine the effective SDS-protein mobility (see eq 1 ) since the two mobilities are of opposite signs. Without EOF, all solute molecules would have migrated faster. The migration of the charged gel matrix under the applied electric field was insignificant, and so, was not considered in eq 1 .

$$
\mu_{\text {eff }}=\mu_{\text {app }}-\mu_{\mathrm{EOF}}
$$

As seen in Table 1, the $10 \% \mathrm{D} / 4 \% \mathrm{~B}$ formulation resulted in the lowest EOF due to the highest viscosity of this gel and the higher ionic strength than the corresponding $10 \% \mathrm{D} / 2 \% \mathrm{~B}$ gel. Overall, EOF mobilities exhibited decreasing tendencies both with increasing dextran (increasing viscosity) and boric acid (increasing ionic strength and cross-linking) concentrations, with a maximum EOF at the lowest composition, i.e., 2\% D/ $2 \%$ B. The measured electroosmotic flow mobility $\left(\mu_{\mathrm{EOF}}\right)$ values were used to correct the apparent electrophoretic mobilities $\left(\mu_{\text {app }}\right)$ of the separated sample components for all interim gel compositions to obtain their effective mobilities $\left(\mu_{\text {eff }}\right.$, eq 1$){ }^{29}$ which relate to the fundamental electromigration through the gel column.

Ferguson Plot Analysis of the Molecular Weight Sizing Proteins. The most commonly used theory to model the electrophoretic migration of polyionic biopolymers, such as SDS-protein complexes, in sieving matrices is based on the Ogston theory. ${ }^{30}$ This model considers that the SDS-protein complexes migrate as nondeformable spherical objects through a porous sieving matrix, resulting in a linear relationship of log mobility $\left(\mu_{\text {eff }}\right)$ vs gel concentration $(T)$ (Ferguson plot $\left.^{31}\right)$, as shown in eq 2 .

$$
\ln \mu_{\mathrm{eff}}=\ln \mu_{\mathrm{eff}, 0}-K_{\mathrm{R}} T
$$

where $\mu_{\text {eff }, 0}$ is the free solution mobility at zero gel concentration and $K_{\mathrm{R}}$ is the retardation coefficient. However, changes in the structure and properties of the sieving polymer over the gel concentration range and/or the charge density or shape (e.g., nonspherical particles) of the various solute molecules over the molecular weight range can lead to a nonlinear relationship, ${ }^{32,33}$ in which case, extension of the Ogston model was suggested. ${ }^{34}$ With increasing gel concentrations, it was assumed that large polyions stretch to a rodlike cylindrical conformation capable of migrating through small pore size sieving matrices. $^{22,35,36}$ In large, multi-subunit protein complexes, such as the Y-shaped IgG molecules, non-rodlike shape selectivity should also be considered.

To examine the fundamental sieving behavior, a series of chemical gel columns were produced with 2.0, 5.0, 7.5, and $10.0 \%$ of the 2 million $M_{\mathrm{W}}$ dextran (D) monomers, each with either the 2.0 or $4.0 \%$ borate (B) cross-linker concentration. A series of molecular weight sieving standards in the range of $20-225 \mathrm{kDa}$ were separated by $\mathrm{CE}$, the effective mobility (corrected for EOF, eq 1) was determined, and Ferguson plots were constructed. As shown in Figure 2, nonlinear concave plots were obtained, with increasing curvature for the highest molecular weight standard $(225 \mathrm{kDa})$ toward the higher monomer concentration gel compositions $(7.5-10 \%)$. It is also seen that the nonlinearity is more severe for the $2 \%$ borate concentration gel than with the $4 \%$ borate gel.

It is reasonable to assume that the charge density and shape (cylindrical) of the molecular weight size standards, assembled from the same polypeptide building blocks with no posttranslational modifications, ${ }^{37}$ are similar. Therefore, the curvature of the plots in Figure 2 likely reflects a change in the chemical gel structure over the range of dextran concentrations. With the lower borate acid-containing gels (2\% B), mostly interchain adducts $(2: 1)$ are formed, and the increasing amount of dextran probably consumes the majority of the added borate at approximately $7.5 \%$ dextran concentration. Consequently, the sieving capability of the $10 \% \mathrm{D}$ gel is not significantly greater as suggested by the heavier curvature of the Ferguson plots in Figure 2A. With the $4 \%$ boric acid-containing gels ( $4 \% \mathrm{~B}$ ), on the other hand, there is still a sufficient amount of borate in the system to cross-link the dextran chains even at a $10 \%$ concentration level, further decreasing the pore size and the effective electrophoretic mobilities of the SDS-laden proteins (Figure 2B). The dotted lines between the two highest dextran concentration matrices (7.5 and 10.0\%) emphasize the slope differences for the 2 and $4 \%$ borate gels. In other words, for the higher concentration 
dextran matrices, the cross-linking will mainly attach separate dextran strands (interchain) into a chemical gel-mesh structure for sieving to occur, and the extent of cross-linking being obviously greater for the $4 \%$ relative to the $2 \%$ boratecontaining composition. On the other hand, both interchain and intrachain borate attachments will more likely occur for the diluted dextran matrix and the mesh size will be larger for the most dilute polymer for a given borate concentration. Moreover, at lower dextran and higher borate cross-linker concentrations, more 1:1 dextran/borate adducts are formed, which could lead to an even larger mesh size due to chain repulsion.

The inherent structural changes as the dextran concentration were varied likely led to the curvatures of the Ferguson plots in Figure 2. In addition, given the high-frequency formation and release of the borate-dextran links, it can be envisioned that the SDS-protein complex may distort the gel matrix as it travels through the mesh, leading to transiently larger mesh pores. The distortion should be the greatest for the highest 225 $\mathrm{k}$ molecular weight standard. Furthermore, distortion should be easier for the $2 \%$ borate gels than for the $4 \%$ B gels, leading to a greater curvature for the former, as seen. It is also important to note that the combination of the borate crosslinked mesh and the large viscosity differences $(\sim 10 \times)$ between the 2 and $10 \%$ dextran gels affect the resulting electrophoretic mobilities.

The nonlinear Ferguson plots demonstrate that the dextran/ borate gel likely undergoes dynamic structural changes as the SDS-proteins electrophorese through the matrix, the extent of change being dependent on the composition of the gel and the size of the protein. Nevertheless, significant sieving is achieved by the borate cross-linked dextran gels, and we next turn to examine the capillary electrophoretic separation of the SDSprotein standards under various gel compositions.

SDS-CGE Separation of the Molecular Weight Sizing Standards. We next explored the actual CE separation of the SDS complexed molecular weight protein standards in borate cross-linked dextran gels using uncoated wall capillary columns. For simplicity again, boundary gel compositions of $10 \% \mathrm{D} / 4 \% \mathrm{~B}, 10 \% \mathrm{D} / 2 \% \mathrm{~B}, 2 \% \mathrm{D} / 4 \% \mathrm{~B}$, and $2 \% \mathrm{D} / 2 \% \mathrm{~B}$ are presented in Figure 3. The separations in the figure reflect the combined effect of molecular sieving and the EOF differences of the various monomer/cross-linker gel compositions, i.e., while more extensive sieving was found with the $10 \% \mathrm{D} / 4 \% \mathrm{~B}$ gel, the migration time window was wider with improved resolution using the $10 \% \mathrm{D} / 2 \% \mathrm{~B}$ composition (Figure 3, traces $\mathrm{a}$ and $\mathrm{b}$ ). This improved separation for the latter gel column was the result of the more than 4.5 times greater countercurrent EOF (Table 1), effectively, acting as a longer column. $^{25,28}$ Similar behavior was observed for the $2 \%$ D gels, in which case shorter migration times were obtained with the higher cross-linker concentration $2 \% \mathrm{D} / 4 \% \mathrm{~B}$ gel (Figure 3, traces $\mathrm{c}$ and $\mathrm{d}$ ). In this instance, while the viscosity of the two formulations did not differ greatly (Table 1), the twofold higher countercurrent EOF of the low borate concentration gel and the likely larger pore size of the $2 \% \mathrm{D} / 4 \% \mathrm{~B}$ gel (extra $1: 1$ borate adduct-mediated repulsion effect) with easier distortion are considered as probable reasons for the more rapid migration, relative to the $2 \% \mathrm{D} / 2 \% \mathrm{~B}$ gel. The separation speed in these two low dextran concentration formulations was higher, due to their approximately 10 times lower viscosities, considering the inverse relationship between the electrophoretic mobility and viscosity. ${ }^{29}$ This suggests the option of

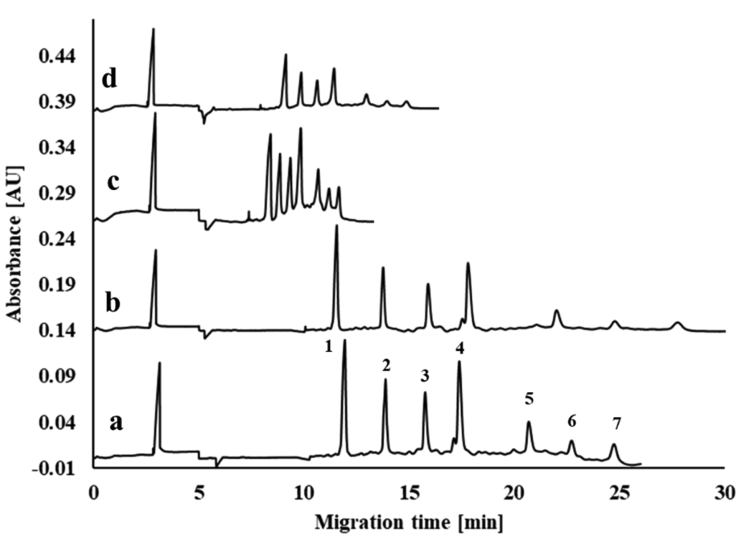

Figure 3. Capillary SDS gel electrophoresis separation of the protein MW sizing ladder at the four boundary gel compositions. (a) $10 \%$ dextran $/ 4 \%$ borate, (b) $10 \%$ dextran $/ 2 \%$ borate, (c) $2 \%$ dextran $/ 4 \%$ borate, and (d) $2 \%$ dextran $/ 2 \%$ borate. Peaks: $1-10 \mathrm{kDa}$ internal standard, 2-20 kDa, 3-35 kDa, 4-50 kDa, 5-100 kDa, 6-150 $\mathrm{kDa}$, and $7-225 \mathrm{kDa}$ sizing standards. Conditions: bare fused-silica capillary $20 \mathrm{~cm}$ (effective length, $30 \mathrm{~cm}$ total, $50 \mu \mathrm{m}$ ID); UV detection at $214 \mathrm{~nm} ; E=500 \mathrm{~V} / \mathrm{cm}$ in a reversed polarity mode (cathode at the injection side), 20 psi pressure from both sides during the separation step at $25^{\circ} \mathrm{C}$; and electrokinetic injection: $5 \mathrm{kV}$ for 10 s.

separation fine tuning by adjusting such parameters as EOF and viscosity.

It is interesting to note that we examined the separation of the molecular weight standards in entangled dextran polymer matrices using Tris-Ches buffer (no borate) and found poorer effective sieving with longer migration times for the same \% D compositions (data not shown), in agreement with the observation of others. ${ }^{38}$ This emphasized the remarkable effect of borate to affect the physical structure of the separation matrix. It is well known that cross-linking of long polymer chains converts entangled polymers into gels. ${ }^{20}$ The relatively poor separation ability of the no borate-containing entangled dextran polymer can be contrasted to linear polyacrylamide, which achieves very high column efficiencies. ${ }^{4}$ The interchain hydrogen bonding in the case of dextran will likely make its polymer chains much more rigid than those of polyacrylamide, in which latter case, increased flexibility is expected allowing a more uniform structure. However, borate cross-linkingmediated stabilization of the dextran chains led to more structured reticulation.

Returning to the dextran-borate matrix, Figure 4 presents the molecular weight calibration plots (apparent mobility vs $\log \mathrm{MW}$ ) for the protein standards for the four boundary gel compositions. The plots for the $10 \%$ dextran gels with 2 and $4 \%$ borate intersect each other at approximately $35 \mathrm{kDa}$ (deeper slope for the $10 \% \mathrm{D} / 2 \% \mathrm{~B}$ gel) that was also observed in the corresponding migration time windows in Figure 3 (traces a and b). This behavior is a reflection of the interplay between the larger EOF of the $10 \% \mathrm{D} / 2 \% \mathrm{~B}$ gel and its decreased mesh, relative to the corresponding higher crosslinked (4\% B) gel. Interestingly, the mobility differences between the 2 and $4 \%$ borate compositions is greater for the $2 \%$ dextran gels than for the $10 \%$ gels. This may be a reflection of the greater charge repulsion due to the negative borate charges on the dextran fibers and the consequent larger mesh size differences for the dilute gels. 


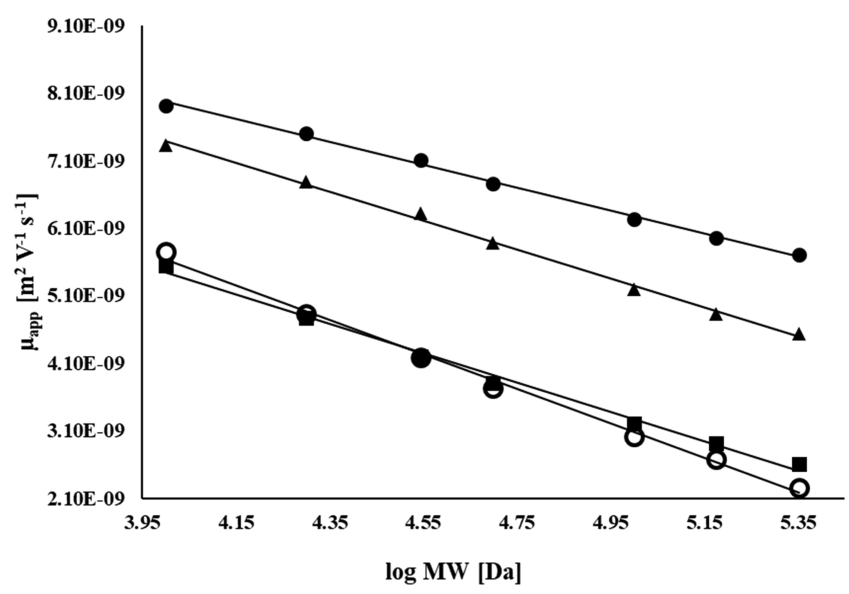

Figure 4. Molecular weight calibration plots of apparent electrophoretic mobility vs log MW for the protein SDS-MW size standards for the four boundary condition gel compositions of $(\boldsymbol{D}) 10 \% \mathrm{D} / 4 \%$ $\mathrm{B},(\mathrm{o}) 10 \% \mathrm{D} / 2 \% \mathrm{~B},(\boldsymbol{\Delta}) 2 \% \mathrm{D} / 2 \% \mathrm{~B}$, and (-) $2 \% \mathrm{D} / 4 \% \mathrm{~B}$.

To more closely examine the separation performance of the various gel compositions, Table 2 summarizes the resolution $\left(R_{\mathrm{s}}\right)$ and selectivity $(\alpha$, both apparent and EOF-corrected effective) values for the $20 \mathrm{kDa} / 50 \mathrm{kDa}$ protein standard pair, as well as for the $50 \mathrm{kDa} / 150 \mathrm{kDa}$ and $150 \mathrm{kDa} / 225 \mathrm{kDa}$ pairs at all four boundary monomer/cross-linker ratios. The first two standard protein pairs were chosen as being the closest in size to the light $(24 \mathrm{kDa})$ and heavy $(49 \mathrm{kDa})$ chain subunits as well as the intact $(\sim 149 \mathrm{kDa})$ monoclonal antibody test sample components shown later in this study. The corresponding data for the $225 \mathrm{kDa}$ size standard is also shown for comparative purposes.

As seen in Table 2, for the protein size pairs shown, the $10 \%$ $\mathrm{D} / 2 \% \mathrm{~B}$ gel composition exhibited the highest resolution and apparent selectivity values, but, the longest separation time. Note that the significant countercurrent EOF in this case (Table 1) contributed to greater apparent selectivities $\left(\alpha_{\text {app }}\right)^{28}$ especially in the higher MW solute range, while the effective selectivity was the highest with the $10 \%$ dextran/4\% borate composition, except for the $150 / 225 \mathrm{kDa}$ pair. The electroosmotic flow may strongly impact the separation of larger proteins as the EOF remains constant, while the mobility of the proteins decreases with increasing MW. The smaller differences between the apparent and effective selectivity values for the $4 \%$ borate-containing gel compositions in contrast to the $2 \% \mathrm{~B}$ gels suggest greater sieving selectivity in the former gels and enhanced EOF effect (longer virtual column) in the latter, contributing to the resolutions obtained.

Analysis of a Therapeutic Monoclonal Antibody and Its Subunits. Having examined the basic sieving behavior for protein standards on the various dextran/borate gel columns, we now turn to the separation of a therapeutic monoclonal antibody and its subunits. While CE analysis of mAbs is widely conducted on commercial D/B columns, examination of the intact $\mathrm{mAb}$ and its subunits, in comparison to the protein standards of comparable molecular weight, can provide further understanding of migration and separation of species in borate cross-linked dextran gels. The SDS-mAb and its subunits, even with close molecular weights, have different cross-sectional areas than the comparable SDS-standards, especially when they possess post-translational modifications such as glycosylation. $^{39}$

A mixture of the SDS complexes of an intact monoclonal antibody, its heavy (HC) and light chain (LC) subunits, and the nonglycosylated heavy chain (ngHC) was selected for this study. Ferguson plots were analyzed, and the results are shown in Figure 5. Here again, concave plots were observed with increasing curvatures toward higher molecular weights and lower borate cross-linker concentrations, similar to that found in Figure 2. Likewise, improved sieving was obtained with higher dextran concentrations for these sample components. The plots corresponding to the nonglycosylated heavy chain (ngHC) and the glycosylated heavy-chain (HC) subunits were apparently parallel with both cross-linker compositions. This behavior is characteristic for components with very similar molecular weights but different mobilities, ${ }^{40}$ in this case due to their shape differences. Note that the charge differences between the nonglycosylated and glycosylated heavy chains will not be high because of borate complexation with glycosylated species ${ }^{39}$ and the low level of glycosylation relative to the rest of the heavy-chain molecule.

Figure 6 shows the separation of the four mAb species using the four boundary gel composition columns. The inset depicts the structural (shape) interpretation of the sample molecules. The migration time shifts shown in the figure are similar to those observed in Figure 3, again, due to the interplay among

Table 2. Resolution $\left(R_{\mathrm{s}}\right)$ and Selectivity ( $\alpha$, Apparent and EOF-Corrected Effective) Values for the $20 \mathrm{kDa} / 50 \mathrm{kDa}, 50 \mathrm{kDa} /$ $150 \mathrm{kDa}$, and $150 \mathrm{kDa} / 225 \mathrm{kDa}$ SDS-MW Protein Size Standard Pairs at the Boundary Monomer/Cross-Linker Concentration Gel Compositions of $10 \%$ Dextran/2\% Borate, 10\% Dextran/4\% Borate, 2\% Dextran/2\% Borate, and 2\% Dextran/4\% Borate ${ }^{a}$

\begin{tabular}{|c|c|c|c|c|}
\hline separation performance & $10 \% \mathrm{D} / 2 \% \mathrm{~B}$ & $10 \% \mathrm{D} / 4 \% \mathrm{~B}$ & $2 \% \mathrm{D} / 2 \% \mathrm{~B}$ & $2 \% \mathrm{D} / 4 \% \mathrm{~B}$ \\
\hline$R_{\mathrm{s}}(20 / 50)$ & 9.33 & 8.08 & 4.94 & 2.99 \\
\hline migration time $[\mathrm{min}] 50 \mathrm{kDa}$ & 17.9 & 17.5 & 11.3 & 9.9 \\
\hline$\alpha_{\text {app }(50 / 20)}$ & 1.28 & 1.26 & 1.16 & 1.12 \\
\hline$\alpha_{\text {eff }(50 / 20)}$ & 1.21 & 1.24 & 1.09 & 1.09 \\
\hline$R_{\mathrm{s}}(50 / 150)$ & 12.05 & 10.97 & 5.94 & 3.45 \\
\hline migration time $[\mathrm{min}] 150 \mathrm{kDa}$ & 24.8 & 22.9 & 13.8 & 11.2 \\
\hline$\alpha_{\text {app (150/50) }}$ & 1.37 & 1.31 & 1.22 & 1.15 \\
\hline$\alpha_{\text {eff }(150 / 150)}$ & 1.25 & 1.28 & 1.11 & 1.10 \\
\hline$R_{\mathrm{s}}(150 / 225)$ & 4.26 & 3.49 & 1.99 & 1.19 \\
\hline migration time $[\mathrm{min}] 225 \mathrm{kDa}$ & 27.8 & 24.9 & 14.7 & 11.7 \\
\hline$\alpha_{\text {app (225/150) }}$ & 1.12 & 1.09 & 1.07 & 1.04 \\
\hline$\alpha_{\text {eff }(225 / 150)}$ & 1.09 & 1.08 & 1.03 & 1.03 \\
\hline
\end{tabular}

${ }^{a} \mathrm{D}$-dextran and $\mathrm{B}$ - borate. 
A

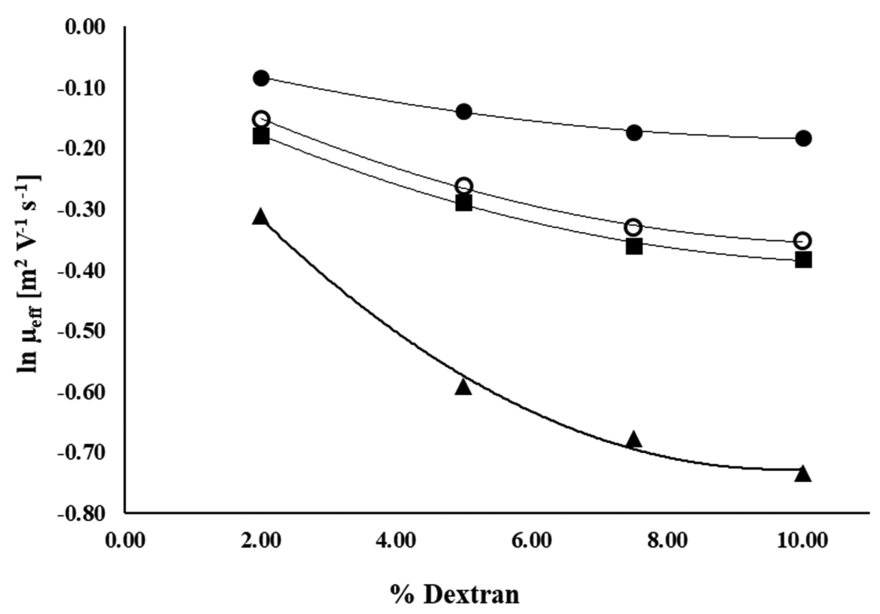

B

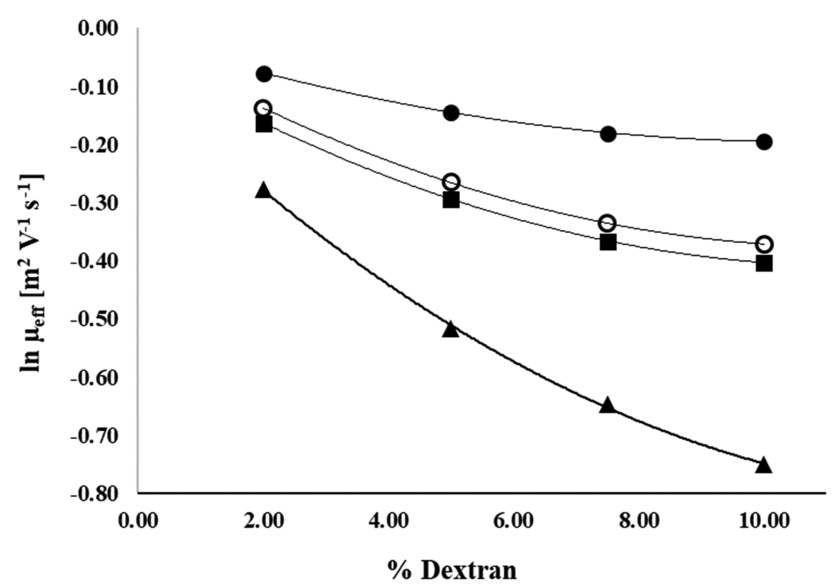

Figure 5. Ferguson plots of the EOF-corrected logarithmic relative electrophoretic mobilities of the monoclonal antibody and its subunits as a function of monomer (dextran) concentration with $2.0 \%$ (A) and $4.0 \%$ (B) boric acid cross-linker. Symbols: $\bullet$ light chain (LC), $\bigcirc$ deglycosylated heavy chain (ngHC), $\mathbf{\square}$ heavy chain (HC), and $\boldsymbol{\Delta}$ intact mAb.

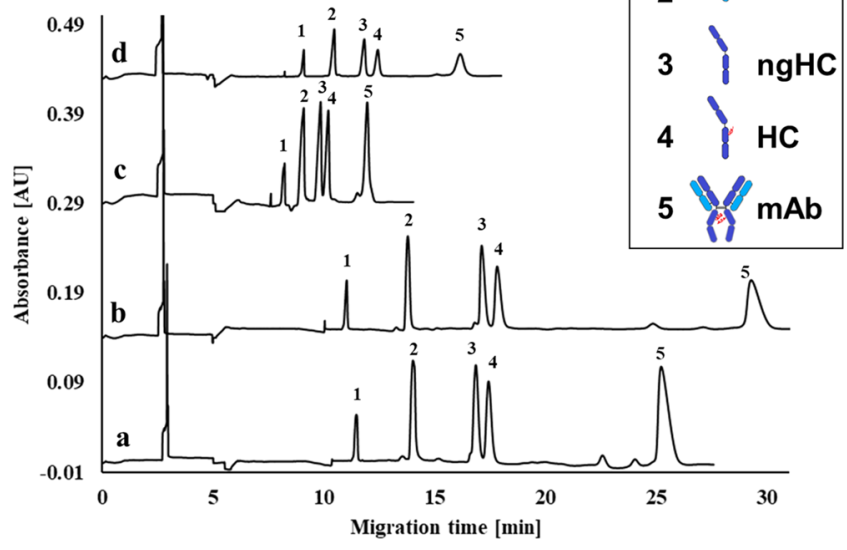

Figure 6. Capillary SDS gel electrophoresis separation of the monoclonal antibody test item and its subunits at the boundary gel compositions. (a) $10 \%$ dextran $/ 4 \%$ borate, (b) $10 \%$ dextran $/ 2 \%$ borate, (c) $2 \%$ dextran $/ 4 \%$ borate, and (d) $2 \%$ dextran $/ 2 \%$ borate. Peaks: 1-10 kDa internal standard, 2-light chain (LC), 3nonglycosylated heavy chain (ngHC), 4-heavy chain ( $\mathrm{HC}$ ), and 5intact monoclonal antibody (mAb). Inset: the structural (shape) interpretation of the sample molecules. Conditions were the same as in Figure 3.

the countercurrent EOF at the different ionic strength buffers, the viscosity, and the extent of cross-linking in the separation matrices. For example, as seen for the protein standards in Figure 3, the fastest separation was obtained with the $2 \% \mathrm{D} /$ $4 \% \mathrm{~B}$ gel. On the other hand, the migration time window was the greatest with the $10 \% \mathrm{D} / 2 \% \mathrm{~B}$ composition. The resolution between the $\mathrm{ngHC}$ and $\mathrm{HC}$ was the best by using the $2 \% \mathrm{D} / 2 \% \mathrm{~B}$ gel (Table 3 ), due to the additional glycanborate-dextran complexation, as reported earlier in ref 39 .

Table 3 lists the resolution and selectivity (both apparent and EOF-corrected effective) values for the $\mathrm{LC} / \mathrm{ngHC}$ and ngHC/HC fragments, as well as the $\mathrm{HC} /$ intact $\mathrm{mAb}$ sample component pairs. The light and the nonglycosylated heavy chains did not possess any glycan moieties, therefore, their separation was considered mostly based on the MW sieving effect in the borate cross-linked dextran matrix. Similar to the analysis of the $20 \mathrm{kDa} / 50 \mathrm{kDa}$ standard protein pair shown above, the $10 \% \mathrm{D} / 2 \% \mathrm{~B}$ gel composition resulted in the highest resolution and apparent selectivity for the $\mathrm{LC} / \mathrm{ngHC}$ pair but again with the longest analysis time. However, the effective (EOF corrected) selectivity was the highest with the standard $10 \%$ dextran/4\% borate concentration gel, again as with the standards.

Separation of the almost similar molecular weight (only $\sim 2.5 \% \mathrm{MW}$ difference) nonglycosylated (ngHC) and regular (HC) heavy chain fragments in all gel compositions was considered the outcome of their shape differences (inset in Figure 6), with the glycosylated form (HC) being bulkier than its less bulky counterpart (ngHC). However, the best separation performance for this $\mathrm{ngHC} / \mathrm{HC}$ pair was obtained with the lowest concentration $(2 \% \mathrm{D} / 2 \% \mathrm{~B})$ monomer/crosslinker composition. The higher $\alpha$-value of the $2 \% \mathrm{D} / 2 \% \mathrm{~B}$, while only $20 \%$ greater than for the $10 \% \mathrm{D} / 2 \% \mathrm{~B}$ gel, was significant since small differences in $\alpha$-values close to unity have a magnified influence on the resulting separation. The observed higher resolution between the glycosylated and nonglycosylated heavy chain fragments by the $2 \% \mathrm{D} / 2 \% \mathrm{~B}$ gel was reported earlier as associated with the larger number of intrachain (1:1) dextran-borate adducts that allowed the formation of dextran-borate-glycoprotein complexes, ${ }^{39}$ increasing selectivity.

Between the $\mathrm{HC}$ and the intact mAb (Table 3), similar to the $50 \mathrm{kDa} / 150 \mathrm{kDa}$ pair of the molecular weight sizing standards (Table 2), the highest resolution was again obtained with the $10 \% \mathrm{D} / 2 \% \mathrm{~B}$ gel. As noted above, the individual sizing components of the MW ladder were assembled from the same polypeptide unit building blocks with no post-translational modification, thus their electromigration behavior was not expected to be the same as that of the similar size $\mathrm{mAb}$ fragments and their intact form, due to the dramatic effect of shape selectivity. Indeed, the intact $\mathrm{mAb}$ migrated at approximately the same time in all compositions as the 225 $\mathrm{kDa}$ SDS-MW standard. The longer migration time (relative to the molecular weight standard) of the intact $\mathrm{mAb}$ in all gel compositions can be explained by its inherent $\mathrm{Y}$ profile (see the inset in Figure 6), and consequently, higher hydrodynamic radius than that of the similar size $150 \mathrm{kDa}$ sizing standard. As 
Table 3. Resolution $\left(R_{s}\right)$ and Selectivity ( $\alpha$, Apparent and EOF-Corrected Effective) Values for the Intact $\mathrm{mAb}$ Test Item and Its Light Chain (LC), Nonglycosylated Heavy Chain (ngHC), and Heavy Chain (HC) Subunits at the Boundary Monomer/ Cross-Linker Concentration Gel Compositions of 10\% Dextran/2\% Borate, 10\% Dextran/4\% Borate, 2\% Dextran/2\% Borate, and $2 \%$ Dextran $/ 4 \%$ Borate $^{a}$

\begin{tabular}{|c|c|c|c|c|}
\hline separation performance & $10 \% \mathrm{D} / 2 \% \mathrm{~B}$ & $10 \% \mathrm{D} / 4 \% \mathrm{~B}$ & $2 \% \mathrm{D} / 2 \% \mathrm{~B}$ & $2 \% \mathrm{D} / 4 \% \mathrm{~B}$ \\
\hline$R_{\mathrm{s}}(\mathrm{LC} / \mathrm{ngHC})$ & 15.18 & 12.37 & 6.46 & 4.6 \\
\hline migration time $[\mathrm{min}] \mathrm{ngHC}$ & 17.2 & 16.9 & 11.9 & 9.8 \\
\hline$\alpha_{\text {app }(\mathrm{ngHC} / \mathrm{LC})}$ & 1.24 & 1.21 & 1.13 & 1.09 \\
\hline$\alpha_{\mathrm{eff}(\mathrm{ngHC} / \mathrm{LC})}$ & 1.18 & 1.19 & 1.07 & 1.06 \\
\hline$R_{\mathrm{s}}(\mathrm{ngHC} / \mathrm{HC})$ & 1.64 & 1.32 & 2.31 & 1.04 \\
\hline migration time $[\mathrm{min}] \mathrm{HC}$ & 17.9 & 17.5 & 12.5 & 10.2 \\
\hline$\alpha_{\text {app (HC/ngHC) }}$ & 1.04 & 1.03 & 1.05 & 1.03 \\
\hline$\alpha_{\text {eff }(\mathrm{HC} / \mathrm{ngHC})}$ & 1.03 & 1.03 & 1.03 & 1.03 \\
\hline$R_{\mathrm{s}}(\mathrm{HC} /$ intact $\mathrm{mAb})$ & 12.81 & 10.25 & 6.81 & 1.49 \\
\hline migration time $[\mathrm{min}]$ intact $\mathrm{mAb}$ & 29.2 & 25.3 & 16.2 & 11.9 \\
\hline$\alpha_{\text {app (intact mAb/HC) }}$ & 1.64 & 1.45 & 1.3 & 1.17 \\
\hline$\alpha_{\text {eff (intact mAb/HC) }}$ & 1.42 & 1.41 & 1.14 & 1.11 \\
\hline
\end{tabular}

Table 4. Shape Selectivity between the HC, ngHC, and Intact mAb and the Corresponding SDS-MW Protein Standards of 50 and $150 \mathrm{kDa}$

$\begin{array}{lcc}\text { shape selectivity } & 10 \% \mathrm{D} / 2 \% \mathrm{~B} & 10 \% \mathrm{D} / 4 \% \mathrm{~B} \\ \alpha_{\text {app }}(50 / \mathrm{HC}) & 1.00 & 1.00 \\ \alpha_{\text {eff }}(50 / \mathrm{HC}) & 1.04 & 1.04 \\ \alpha_{\text {app }}(50 / \mathrm{ngHC}) & 0.96 & 0.97 \\ \alpha_{\text {eff }}(50 / \mathrm{ngHC}) & 1.01 & 1.01 \\ \alpha_{\text {app }}(150 / \text { intact mAb }) & 1.18 & 1.11 \\ \alpha_{\text {eff }}(150 / \text { intact mAb }) & 1.17 & 1.15\end{array}$

shown in Table 4, the shape selectivities between the corresponding size SDS MW sizing standards were similar for the most linear IgG subunits of the nonglycosylated heavy chain $(50 / \mathrm{ngHC})$, somewhat different for the glycosylation possessing $\mathrm{HC}$ due to its bulkier shape because of the carbohydrate side chain $(50 / \mathrm{HC})$ and very different for the Yshaped intact form $(150 / \mathrm{mAb})$ for most gel formulations.

To emphasize the shape selectivity, the molecular weight estimations for the intact monoclonal antibody and its subunits were calculated (Table 5) based on the calibration plots in

Table 5. Molecular Weight Estimation of the Intact IgG and Its Subunits Based on Figure 4, Using the Four Boundary Gel Compositions ${ }^{a}$

$\begin{array}{lcccc}\text { MW (kDa) } & 10 \% \mathrm{D} / 2 \% \mathrm{~B} & 10 \% \mathrm{D} / 4 \% \mathrm{~B} & 2 \% \mathrm{D} / 2 \% \mathrm{~B} & 2 \% \mathrm{D} / 4 \% \mathrm{~B} \\ \mathrm{LC}(24.0) & 20.7 & 21.0 & 30.4 & 23.2 \\ \text { ngHC (48.1) } & 48.2 & 48.9 & 66.5 & 50.2 \\ \mathrm{HC}(49.4) & 55.2 & 56.1 & 89.5 & 68.8 \\ \text { intact mAb } & 204.3 & 194.8 & 337.3 & 252.7\end{array}$

$(149.0)$

${ }^{a}$ The actual MW values of the intact IgG and its subunits are listed in the first column.

Figure 4. The substantial molecular weight overestimation of the intact IgG (actual MW $=149 \mathrm{kDa}$ ) in all cases reflects the shape selectivity of the borate cross-linked dextran gel. The greatest overestimation was observed for the $2 \% \mathrm{D} / 2 \% \mathrm{~B}$ gel. The $10 \%$ D gels slightly underestimated the MW of the light chain and resulted in reasonable values for the nonglycosylated heavy chain fragment.

\section{CONCLUSIONS}

Capillary sodium dodecyl sulfate electrophoresis with borate cross-linked dextran gel is an established platform technology in the biopharmaceutical field for rapid characterization, release, and stability studies of protein therapeutics. This transiently cross-linked gel composition features easy replenishment of the sieving matrix in the narrow-bore capillary, supporting fully automated operation. In this article, physical, electrokinetic, and separation performance characteristics were reported to shed light on the fundamental basis of the method. Utilization of the Ferguson plots allowed the examination of the important details of the separation mechanism of proteins in SDS-CGE with respect to the effect of monomer (dextran) and cross-linker (borate) concentrations. Note that measurable EOF values were observed in all boundary gel compositions, which can affect separation selectivity, i.e., higher countercurrent EOF had the same effect as the use of a longer separation capillary with the same sieving matrix, without the associated higher applied electric field strength requirement. Importantly, high absolute migration time reproducibility can be achieved (RSD $<0.96 \%)$, even with the countercurrent EOF (run-to-run $(n=16)$, column-to-column $(n=8)$, and gelto-gel $(n=3)$, considering all boundary gel compositions), if the careful column washing procedures are applied, as described in the Experimental Section.

Among the four boundary concentration gel compositions shown, the $2 \% \mathrm{D} / 4 \% \mathrm{~B}$ formulation offered the fastest analysis time, while still providing adequate separation performance for the samples analyzed in this study. The highest separation window and MW selectivity were obtained with the $10 \% \mathrm{D} / 2 \%$ $\mathrm{B}$ composition, but, with the longest separation time due to the considerable countercurrent EOF. The $2 \% \mathrm{D} / 2 \% \mathrm{~B}$ matrix 
offered the best resolution between glycosylated and nonglycosylated species by exploiting the potential complexation between the 1:1 dextran/borate adducts and the carbohydrate moiety of the glycoprotein. The $10 \%$ dextran/4\% boric acidcontaining gel showed the best general screening capability, hence represented a good starting gel composition for any design of experiment (DOE) study. The various monomer/ cross-linker concentration ratio gels, thus, offer a broad basis for separation optimization and the selection of the appropriate gel composition depending on the specific separation problem at hand.

\section{AUTHOR INFORMATION}

\section{Corresponding Author}

András Guttman - Csaba Horváth Memorial Laboratory of Bioseparation Sciences, Research Center for Molecular Medicine, Faculty of Medicine, Doctoral School of Molecular Medicine, University of Debrecen, Debrecen H-4032, Hungary; Translational Glycomics Group, Research Institute for Biomolecular and Chemical Engineering, University of Pannonia, Veszprem H-8200, Hungary; ○ orcid.org/00000002-7838-082X; Email: guttman@mik.uni-pannon.hu

\section{Authors}

Csenge Filep - Csaba Horváth Memorial Laboratory of Bioseparation Sciences, Research Center for Molecular Medicine, Faculty of Medicine, Doctoral School of Molecular Medicine, University of Debrecen, Debrecen H-4032, Hungary

Barry L. Karger - Barnett Institute, Northeastern University, Boston, Massachusetts 02115, United States; 이이.org/ 0000-0002-9786-0036

Complete contact information is available at:

https://pubs.acs.org/10.1021/acs.analchem.1c01636

\section{Notes}

The authors declare the following competing financial interest(s): The authors declare no known competing financial interests or personal relationships that could have appeared to influence this work. A patent application has been filed for the gel compositions and method of use.

\section{ACKNOWLEDGMENTS}

The authors gratefully acknowledge the support of the BIONANO_GINOP-2.3.2-15-2016-00017 project and the V4-Korea Joint Research Program, project National Research, Development, and Innovation Office (NKFIH) (NN 127062) grants of the Hungarian Government. This work was supported by the TKP2020-IKA-07 project financed under the 2020-4.1.1-TKP2020 Thematic Excellence Programme by the National Research, Development, and Innovation Fund of Hungary. This work was also supported by the UNKP-20-3-IIDE-294 New National Excellence Program of the Ministry for Innovation and Technology. The stimulating discussions with Profs Ivan Donati (Trieste, Italy) and Tibor Chovan (Veszprem, Hungary) are greatly appreciated. This is contribution \#168 from the Csaba Horváth Memorial Laboratory of Bioseparation Sciences.

\section{ABBREVIATIONS USED}

CGE capillary gel electrophoresis

SDS sodium dodecyl sulfate

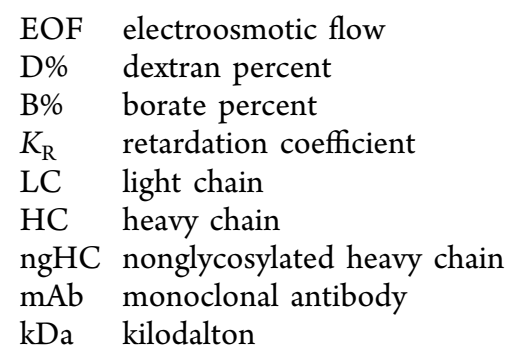

\section{REFERENCES}

(1) Dawod, M.; Arvin, N. E.; Kennedy, R. T. Analyst 2017, 142, $1847-1866$

(2) Toraño, J. S.; Ramautar, R.; de Jong, G. J. Chromatogr. B 2019, $1118-1119,116-136$

(3) Guttman, A. J. Chromatogr. Sci. 2003, 41, 449-459.

(4) Cohen, A. S.; Karger, B. L. J. Chromatogr. A 1987, 397, 409-417.

(5) Ganzler, K.; Greve, K. S.; Cohen, A. S.; Karger, B. L.; Guttman, A.; Cooke, N. C. Anal. Chem. 1992, 64, 2665-2671.

(6) Hunt, G.; Nashabeh, W. Anal. Chem. 1999, 71, 2390-2397.

(7) Benedek, K.; Guttman, A. J. Chromatogr. A 1994, 680, 375-381.

(8) Werner, W. E.; Demorest, D. M.; Stevens, J.; Wiktorowicz, J. E. Anal. Biochem. 1993, 212, 253-258.

(9) Crihfield, C. L.; Holland, L. A. Anal. Chem. 2021, 93, 15371543.

(10) Liu, Y.; Reddy, P.; Ratnayjake, C. K.; Koh, E. V. Methods and compositions for capillary electrophoresis (CE). US Patent US7,831,317 B22003.

(11) Beckman, J.; Song, Y.; Gu, Y.; Voronov, S.; Chennamsetty, N.; Krystek, S.; Mussa, N.; Li, Z. J. Anal. Chem. 2018, 90, 2542-2547.

(12) Sänger-van de Griend, C. E. Electrophoresis 2019, 40, 23612374.

(13) Pezron, E.; Ricard, A.; Leibler, L. J. Polym. Sci., Part B: Polym. Phys. 1990, 28, 2445-2461.

(14) Miyazaki, Y.; Yoshimura, K.; Miura, Y.; Sakashita, H.; Ishimaru, K. Polyhedron 2003, 22, 909-916.

(15) Harris, P. C. J. Pet. Technol. 1993, 45, 264-269.

(16) Witten, T. A.; Cohen, M. H. Macromolecules 1985, 18, 19151918.

(17) Geurink, L.; van Tricht, E.; Dudink, J.; Pajic, B.; Sänger-van de Griend, C. E. Electrophoresis 2021, 42, 10-18.

(18) Szigeti, M.; Guttman, A. Mol. Cell. Proteomics 2019, 18, 25242531.

(19) Allmendinger, A.; Dieu, L. H.; Fischer, S.; Mueller, R.; Mahler, H. C.; Huwyler, J. J. Pharm. Biomed. Anal. 2014, 99, 51-58.

(20) Maitra, J.; Shukla, V. K. J. Polym. Sci. 2014, 4, 25-31.

(21) Deuel, H.; Neukom, H. Makromol. Chem. 1949, 3, 113-126.

(22) Filep, C.; Guttman, A. Anal. Chem. 2020, 92, 4023-4028.

(23) Yin, H. F.; Lux, J. A.; Schomburg, G. J. High Resolut. Chromatogr. 1990, 13, 624-627.

(24) Sacco, P.; Furlani, F.; Paoletti, S.; Donati, I. Biomacromolecules 2019, 20, 3070-3075.

(25) Stellwagen, N. C.; Stellwagen, E. J. Chromatogr. A 2009, 1216, 1917-1929.

(26) Sacco, P.; Furlani, F.; Cok, M.; Travan, A.; Borgogna, M.; Marsich, E.; Paoletti, S.; Donati, I. Biomacromolecules 2017, 18, 42064213.

(27) Cheng, J.; Mitchelson, K. R. Anal. Chem. 1994, 66, 4210-4214.

(28) Kenndler, E. J. Capillary Electrophor. 1996, 3, 191-198.

(29) Grossman, P. D.; Colburn, J. C.; Lauer, H. H. Anal. Biochem. 1989, 179, 28-33.

(30) Ogston, A. G. Trans. Faraday Soc. 1958, 54, 1754-1757.

(31) Ferguson, K. A. Metabolism 1964, 13, 985-1002.

(32) Bryan, J. K. Anal. Biochem. 1977, 78, 513-519.

(33) Rodbard, D.; Chrambach, A. Proc. Natl. Acad. Sci. U.S.A. 1970, 65, 970-977.

(34) Tietz, D. J. Chromatogr. B: Biomed. Sci. Appl. 1987, 418, 305344. 
(35) Barron, A. E.; Blanch, H. W.; Soane, D. S. Electrophoresis 1994, $15,597-615$.

(36) Lumpkin, O. J.; Dejardin, P.; Zimm, B. H. Biopolymers 1985, 24, 1573-1593.

(37) Millipore, E. Protein Markers 10-225 kDa; BIO-69079, 2019.

(38) Mitchelson, K. R.; Cheng, J. Capillary Electrophoresis with Glycerol as an Additive. In Capillary Electrophoresis of Nucleic Acids; Springer, 2001; Vol. 162, pp 259-277.

(39) Filep, C.; Guttman, A. Anal. Chem. 2021, 93, 3535-3541.

(40) Andrews, A. T. Electrophoresis, Theory, Techniques and Biochemical and Clinical Applications, 2nd ed.; Claredon Press Oxford: Oxford, England, 1986. 